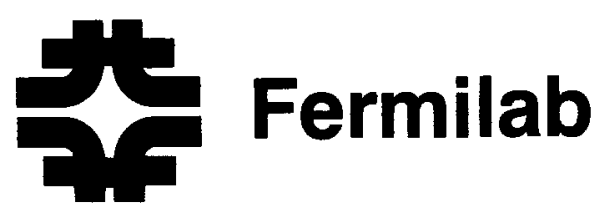

TM-1315

0102.000

SSC-19

\title{
SSC TEST LATTICES
}

E. D. Courant, D. R. Douglas, A. A. Garren, and D. E. Johnson April 20, 1985 
SSC TEST LATTICES

E.D.Courant, D.R.Douglas, A.A.Garren and D.E.Johnson

\subsection{INTRODUCTION}

A set of eight test lattices for the SSC have been devised for such purposes as the investigation of the dependences of chromatic properties and dynamic aperture on the type, field, physical aperture and errors of the magnets, on the sextupole correction scheme, on the tunes and on the cell phase advances. They are distinguished from realistic lattices in that certain features of the latter are missing - most notably the crossing magnets that bring the two counter-rotating proton beams into collision at the interaction points, and the utility insertions, which are the sites for the injection, beam abort, and radiofrequency systems. Furthermore the placement of magnets in the cells is simplified.

There were several reasons for these approximations. First, they simplified the computations so that it was possible to devise lattices that spanned the magnetic fields and cell phase advances under consideration quickly, setting the stage for parallel tracking studies. Second, it was possible to impose a high degree of similarity on the lattices, which is important so that performance differences arising from magnetic fields and errors not be confused by structural differences between the lattices. Third, the lattices could be designed with a relativily high periodicity and degree of symmetry. Thus, effects of field imperfections would not be obscured by an unfortunate choice of periodicity or symmetry. Fourth and last, the test lattices may serve as a yardstick, when compared to realistic lattices with lower periodicity and less symmetry, to assess the weight that should be given to these factors.

Despite these simplifications, we believe that calculations based upon these test lattices are relevant to realistic ones. The slight changes in focussing and dispersion due to the crossing dipoles should have little effect. The utility insertions do reduce the periodicity; however their contribution to the chromaticity is small compared to that of the crossing insertions.

One feature not present in previous SSC lattice designs [1-5] has been included, namely special matching sections called phase trombones whose phase advances can be altered to compensate for changes in the beta functions at the crossings, or to alter the tunes.

In the balance of this paper the lattices will be described, and chromatic properties shown for two different sextupole distributions. 


\subsection{STRUCTURAL DESCRIPTION OF THE LATTICES}

In this section we will describe the structure of the superperiods and of their components. All of the lattices have the same overall structure, but differ in their magnetic fields and cell betatron phase advances. The parameters distinguishing the test lattices will be given in the next section, while here their common features will be emphasized.

\subsection{Superperiod structure}

The test lattices have six superperiods, each containing four components: $A, D, T, X$ and their antisymmetric reflections $A^{\sim}, D^{\sim}, T^{*}, X^{\sim}$. The meaning of an antisymmetric reflected beamline is to place its magnets and drifts in mirror reflected sequence and to reverse the signs of the gradients. The complete superperiod $\mathbf{S}$ may then be written symbolically as

$$
\mathbf{S}=A^{\sim} A \quad D \quad T \quad X \quad X^{\sim} \quad T^{\sim} D^{\sim}
$$

The components of the superperiod are the following:

$A \sim A$ - - the arc of regular FODO type bending cells

$D, D^{\sim}---$ dispersion suppressors

T, $T^{\sim}$-- phase shifting 'trombones'

$X X^{\sim}---$ the low beta crossing insertion

The superperiod has two antisymmetric reflection points, one at the arc center and the other at the collision point. A schematic diagram of the superperiod is shown in Fig. 1 .

\subsection{Motivation For The Antisymmetric Structure}

The explanation for the antisymmetric structure is that there are two proton rings. These are parallel except in the vicinity of the collision points, where the beams cross at a very small angle, pass through common quadrupole triplets, are split by common dipoles, and bent back to their separate paraliel beam lines. The common quadrupoles have equal and opposite focussing effects on the two beams, so it would be extremely awkward if the separated quadrupoles that follow did not also have opposite focussing on the two beams. Therefore all adjacent quadrupoles on the two rings are given opposite polarity. 
Now consider one beam as it traverses the crossing insertion. If the focussing is symmetric about the interaction point (I.P.), then the beta functions of the two beams, and the phases of the insertion will be different. In addition the periodicity will be lowered and the sextupole corrections complicated. Therefore, while not absolutely essential, antisymmetry about the crossing points simplifies the lattice of a proton-proton collider considerably.

The test lattices also have antisymmetry about the arc centers, and an odd number of half-cells, which is required for six superperiods. However rings with three superperiods and symmetry about the arc centers are also reasonable and have also been used in previous SSC and CBA designs.

\section{$2.3 \quad$ Arcs}

We turn now to a discussion of the components of the superperiod. Most of the superperiod's length is taken up by the arc $A^{\sim A}$ of regular FODO bending cells. The arc contains a half-integral number of cells, Narc $=n+1 / 2$, where $n$ is a multiple of 6 or 4 for 60 and 90 degree cells respectivily. As a result the chromaticity sextupoles repeat in integral numbers of betatron wavelengths. This scheme results in a partial cancellation of geometric effects.

\subsubsection{Regular Cells -}

Fig. 2 shows the lattice and beta functions of a single cell. The bending magnets are represented as a single dipole slot. It is a simple matter to subdivide them into whatever lengths the magnet designers find expedient, or at whatever intervals are suitable for application of thin lens multipoles in the tracking studies. The dipoles are of the wedge variety, with no edge focussing.

Two further simplifications have been made in the cells. The dipole slots are centered between the quadrupoles, and both sextupoles are placed in the same half-cell, following a suggestion by $A$. Chao. These simplifications permit antisymmetry about the arc center without the introduction of a discontinuity in either the bending or the sextupoles. 


\subsection{Dispersion Suppressors}

The dispersion suppressors $D$ and $D^{\sim}$ border the arc (Fig. 1). Each consists of two cells with reduced total bending angle equal to that of one cell. The cell and quadrupole lengths and gradients are the same as those of regular cells. The scheme works identically on either $D$ or $D^{\sim}$. In the real collider the adjacent suppressors on the two beams are $D$ and $D^{\sim}$, so that the dipole placement remains the same for the two beamlines.

If the two cells of $D$ as one approaches the I.P. are called $C 1$ and $C 2$, dipoles of length $(1-a) * L b$ and $a * L b$ are placed in the half-cells of $\mathrm{Cl}$ and $\mathrm{C} 2$ respectivily, where $\mathrm{Lb}$ is the length of the dipole. It can be shown that the initial dispersion vector is brought identically to zero by $D$ if $a$ has the value

$$
a=1 /[2(1-\cos \mu)] \text {, }
$$

where $M$ is the phase advance per cell. Thus the bending in $\mathrm{C} 1$ and $\mathrm{C} 2$ is 0 and 1 for 60 degree cells and $1 / 2$ and $1 / 2$ for 90 degree cells, compared to a regular cell. These results have been given previously by R. Helm [6].

The method applies also if the dipoles are not centered, so long as the centers of gravity of the dipoles are placed at the same locations in the half-cells of $\mathrm{Cl}$ and $C 2$ as they have in the normal cells. Fig. 3 shows the dispersion suppressor $D$ of a 60 degree test lattice.

Since the focussing of the suppressors is virtually identical to that of regular cells, the normal cell beta values are mapped from their entrance to their exit.

\subsection{Phase Trombones}

The phase trombone $T$, shown in Fig. 4, follows the dispersion suppressor $D$, so it is dispersion free. The trombone closiy resembles three regular cells without dipoles. It contains seven quadrupoles powered symmetrically about its center, so that there are four independent circuits. These are used to map the normal cell beta and alpha values at its entrance to a waist at its center with zero values of the alphas, so that the entrance values of the betatron functions are repeated at the end of the trombone. Two additional constraints are that the horizontal and vertical phase advances should equal whatever values are required to produce the desired overall tunes of the ring. 
By using these trombones, it is possible to change the beta function at the I.P. from one meter to several hundred meters while keeping the tunes constant, or to change the tunes by a few units.

It should be noted that the horizontal and vertical phase advances through $T$ and $T^{\sim}$ are interchanged, so that their combined effect is to move the tune along the diagonal. For the most part this is what is needed, since the antisymmetry of the crossing insertion $X^{\sim}$ assures that it will always produce equal phases. To split the tunes, it will be necessary to power the four circuits of $T$ and $T^{\sim}$ independently, which will break the antisymmetry and the equal and opposite strength of adjacent quadrupoles on the two beamlines, to some degree.

\subsection{Crossing Insertion}

The left half $X$ of the crossing insertion $X X^{\sim}$ is shown in Fig. 5. It follows the dispersion suppressor $D$ and the phase trombone $T$, which in turn follow the arc. Consequently, the beta and alpha values of the regular cells, in the center of a QF quadrupole, are carried to the entrance of $X$ on the left, but the dispersion and its slope are zero there. The insertion, 500 meters long, is nearly identical to that of the SSC Reference Design A [4], except that the crossing magnets $B+$ and $B-$, located at the ends of the space between $Q 3$ and $Q 4$ are left out. $X$ consists of two quadrupole triplets, though $Q 1$ and $Q 2$ are each split into two physical magnets. The six quadrupoles are adjusted to produce a beam waist with zero values of the alphas and the required value of betat at the collision point (I.P.), adjust the phases and limit the maximum beta values in the quadrupoles. The horizontal and vertical betas at the I.P. must be the same so that the right side of the insertion, $\mathrm{X}^{\sim}$, will produce the same beta functions at its right end as occur at the center of the $Q D$ quadrupole of a normal cell. In the experimental configuration, betat=1 meter, and the total phase advance of the insertion is one tune unit.

\subsection{Number of Cells And Tune Values}

A quantization in the cell number is imposed by global constraints. As mentioned above, the number of cells in an arc, Narc, should be one half-cell more or less than a multiple of 6 or 4 for 60 or 90 degree cells respectively, in order that the sextupoles fill an integral number of betatron wavelengths. If this rule is violated, as it is in one of the lattices, then a few half-cells at the ends of the arc should not have sextupoles. 
Second, it was expedient to set the phase advance of the low-beta insertion equal to unity. This was the value required for the Reference Designs [4].

Third, it was decided that in the low beta configuration the phase of the trombone should be close to that of three normal cells, so that its chromatic contribution is minimized.

Last, in view of the six-fold periodicity, tune values of about $6 \mathrm{k}+2.3$ or $6 \mathrm{k}+4.3$ were chosen, that is between one and two units from a half integer structure resonance.

Application of the above constraints leads to certain allowed values of the number of cells in the arcs. In order to obtain 360 degrees of total bending, some adjustments in the length or strength of the dipoles are needed.

\subsection{SPECIFIC DESCRIPTION OF THE LATTICES}

There are eight lattices, corresponding to four magnetic fields and two cell phase advances, 60 and 90 degrees. Six of the lattices were designed in December 1984 for 6.5, 5, and 3 tesla magnets. Subsequently it was decided to concentrate the future high-field magnet development on a 6 tesla magnet. Hence two additional lattices have recently been designed corresponding to 6 tesla. Since considerable tracking studies have already been done on the 6.5 and 5 tesla lattices, they are included in this paper. One expects the properties of the 6 and 6.5 tesla machines to be very similar.

\subsection{Cells}

The main parameter to be chosen was the length $L$ of the half-cell. It was decided to choose $L$ according to a simple recipe, namely that $L \theta=0.8$, where $\theta$ is the bending angle in radians per half cell. This rule equalizes the dispersion, so that a given momentum deviation will always cause the protons to see the same strength of multipole fields. The value is close to that of existing machines and was the value used in the SSC Reference Design.

The magnetic field of the single dipole slot was chosen to give the same bend angle as would eight separate dipoles with 0.5 meter separation at the nominal fields (except for the $C$ lattices, which have ten dipoles).

The quadrupole gradient in the cells was set between 130 and 150 tesla/meter, as it was in the Reference Designs. However the quadrupole gradients near the crossings were set 
as high as $300 \mathrm{~T} / \mathrm{m}$ in order to limit the maximum beta values.

Besides the dipole slots and quadrupoles, there are two drift spaces in each half-cell for sextupoles, correction elements, pumps, etc. We have taken their combined length to be about 10 meters.

\subsection{Parameters}

By following the principles set forth in sections 2 and 3. the eight test lattices were designed, using the SYNCH program. Their principal parameters are given in Tables I and II. 


\subsection{CHROMATIC EFFECTS}

The variations of the tunes and the beta values at the I.P. with momentum deviation have been explored using the programs SYNCH and DIMAT, which give good agreement.

Fig. 6 shows the tune variations of the eight lattices for the two family scheme having an $F$ and a $D$ sextupole in each cell. These variations appear to be acceptable over the expected operating range of $d p / p= \pm 0.004$. The corresponding variations of betat are shown in Fig. 7 .

A simple non-interleaved scheme, in which two F sextupoles are placed 180 degrees apart in phase, followed by two $D$ sextupoles also separated by 180 degrees, was also investigated. Figs. 8 and 9 show the tune and betat variations for two of the lattices with the non-interleaved scheme. The flat portion of the tune curve is extended, particularily for the 3 tesla lattices. This is probobly due to the larger number of cells for these lattices; hence the sextupole strengths do not become too high. Since the physical aperture corresponds to only about $\pm 0.5 \%$ in momentum, the difference may be irrelevant.

So far several of the lattices have been tracked with the interleaved scheme, and one with the non-interleaved one. For this case at least, the latter scheme gives a much larger dynamic aperture than the former [7].

\subsection{CONCLUSIONS}

A set of Test Lattices designed with varying magnetic fields and betatron phase advances has been constructed, and shown to be useful for studies of chromatic correction and dynamic aperture.

\subsection{ACKNOWLEGEMENTS}

We wish to thank the other members of Group A of the SSC Aperture Workshop of October 1984 and the participants in a meeting on lattice issues at BNL in December 1984 for their suggestions. We are also very grateful to M.J. Syphers for editorial assistance. 


\subsection{REFERENCES}

[1] A. Garren, "20 TeV Collider Lattices with Low-Beta Insertions", Proceedings of the 12th International Conference on High-Energy Accelerators, Fermilab, August 1983.

[2] A. Garren, "A 6.5 Tesla Lattice Example", Accelerator Physics Issues for a Superconducting Super Collider, University of Michigan Report UM HE 84-1, December 1983,.

[3] D. Neuffer, "A Lattice for a 'Low-Field' Superconducting Super Collider" Accelerator Physics Issues for a Superconducting Super Collider, University of Michigan Report UM HE 84-1, December 1983.

[4] Superconducting Super Collider, Reference Designs Study for U.S. Department of Energy, May 8, 1984.

[5] R. Diebold and D.E. Johnson, Summary Report of the pp Interaction Regions Group, Proceedings of the 1984 Summer Study on the Design and Utilization of the Superconducting Super Collider, Snomass, 1984.

[6] R. H. Helm, "Note on Reversible Dispersion Matching with Arbitrary Phase Advance". Accelerator Physics Issues for a Superconducting Super Collider, University of Michigan Report UM HE 84-1, December 1983.

[7] D. Douglas, E. Forest, Private Communication. 
Table I --- Cell Parameters of the Test Lattices

\begin{tabular}{|c|c|c|c|c|c|c|c|c|}
\hline Case & $\begin{array}{l}\text { Phase } \\
\text { deg }\end{array}$ & $\begin{array}{l}\text { Field } \\
\text { tesla }\end{array}$ & $\begin{array}{l}\text { Bslot } \\
\text { tesla }\end{array}$ & $\begin{array}{r}\text { Gradient } \\
\text { tesla/m }\end{array}$ & $\underset{\mathrm{m}}{\mathrm{Lcell}}$ & $\underset{m}{\text { Lslot }}$ & $\begin{array}{l}\text { Lquad } \\
\mathbf{m}\end{array}$ & $\underset{\mathbf{m}}{\mathrm{L} \operatorname{cor} r}$ \\
\hline Al & 60 & 6.469 & 6.198 & 135.7 & 200 & 83.5 & 5.0 & 5.75 \\
\hline A2 & 90 & 6.566 & 6.291 & 138.0 & 200 & 83.5 & 7.0 & 4.75 \\
\hline DI & 60 & 5.994 & 5.748 & 135.7 & 200 & 85.0 & 5.0 & 5.00 \\
\hline D2 & 90 & 6.006 & 5.751 & 134.6 & 205 & 86.0 & 7.0 & 4.75 \\
\hline Bl & 60 & 5.096 & 4.910 & 136.6 & 220 & 95.5 & 4.5 & 5.00 \\
\hline B2 & 90 & 5.096 & 4.910 & 145.6 & 220 & 95.5 & 6.0 & 4.25 \\
\hline $\mathrm{Cl}$ & 60 & 3.054 & 2.948 & 129.8 & 290 & 129.5 & 3.5 & 6.00 \\
\hline C2 & 90 & 2.989 & 2.885 & 129.0 & 290 & 129.5 & 5.0 & 5.25 \\
\hline
\end{tabular}

Table II --- Cell Numbers and Orbit Properties of the Test Lattices

Case Ncells Narc Circum- Tune Beta Eta Beta Chrom-

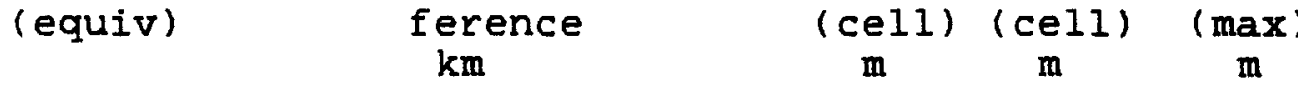

$\begin{array}{llrrrrrrr}\text { A1 } & 405 & 65.5 & 93.60 & 82.29 & 345 & 3.87 & 4627 & -200 \\ \text { A2 } & 399 & 64.5 & 92.40 & 118.27 & 339 & 2.13 & 4183 & -260 \\ \text { D1 } & 429 & 69.5 & 98.40 & 85.35 & 345 & 3.66 & 3910 & -205 \\ \text { D2 } & 423 & 68.5 & 99.56 & 124.35 & 347 & 2.06 & 3900 & -267 \\ \text { B1 } & 447 & 72.5 & 111.90 & 88.30 & 380 & 3.86 & 4415 & -206 \\ \text { B2 } & 447 & 72.5 & 111.90 & 130.29 & 373 & 2.09 & 4187 & -275 \\ \text { C1 } & 549 & 89.5 & 181.49 & 106.29 & 512 & 4.19 & 4625 & -227 \\ \text { C2 } & 561 & 91.5 & 181.49 & 157.29 & 503 & 2.27 & 4454 & -308\end{array}$




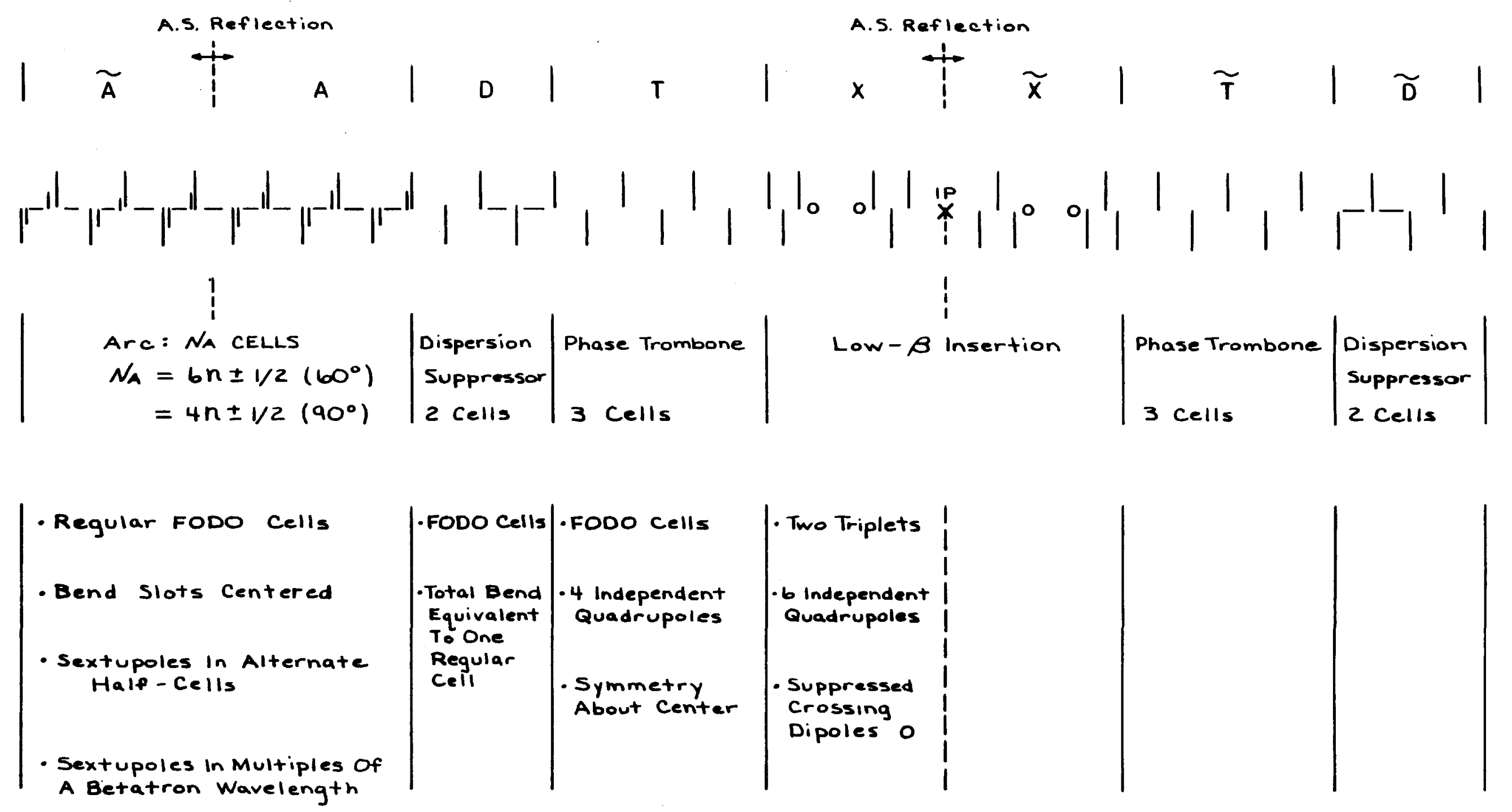




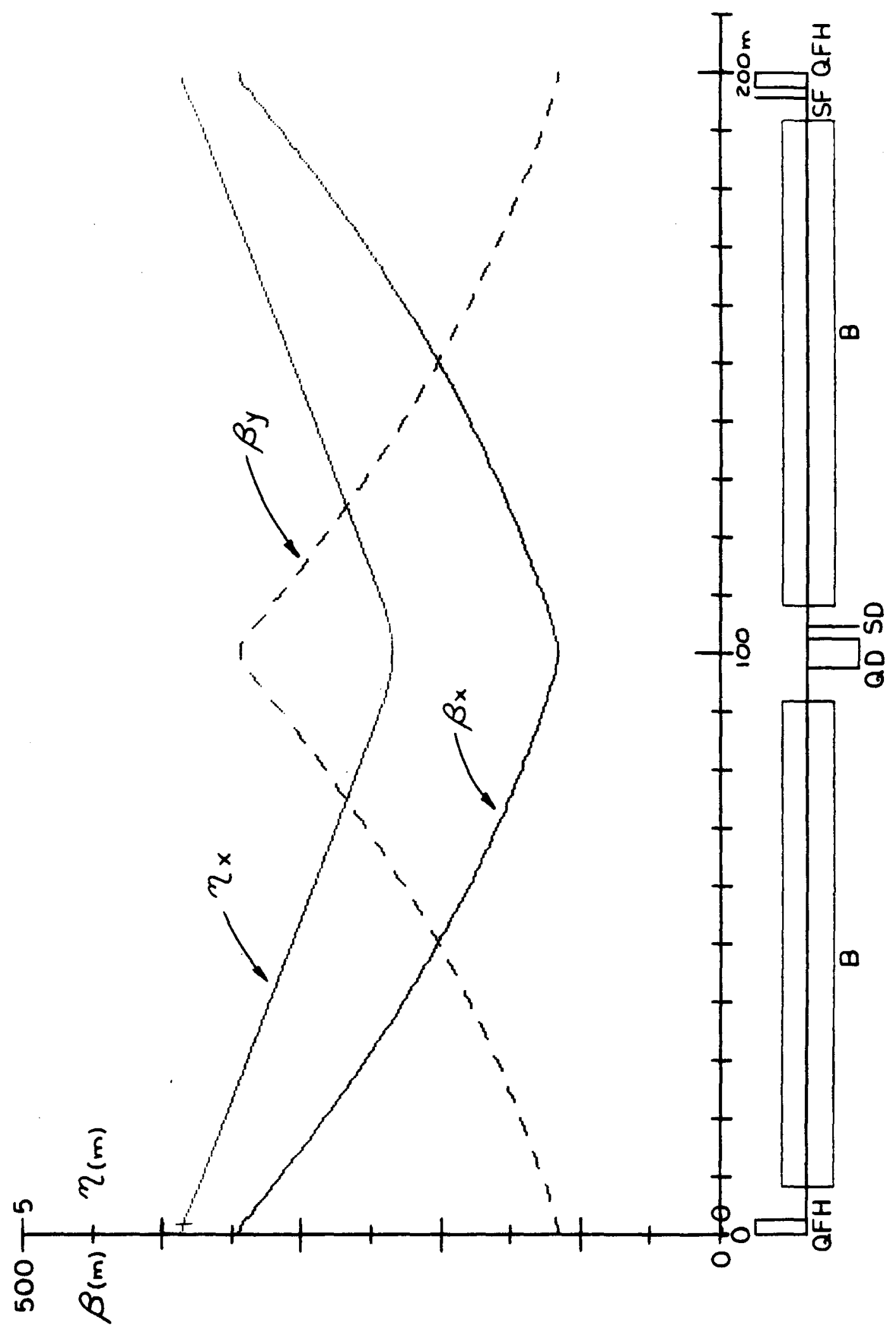

$\bar{u}$
$j$
$\frac{1}{\sigma}$
$\frac{J}{\sigma}$
$\dot{\square}$
$N$
$\dot{\sigma}$
$\frac{1}{4}$ 


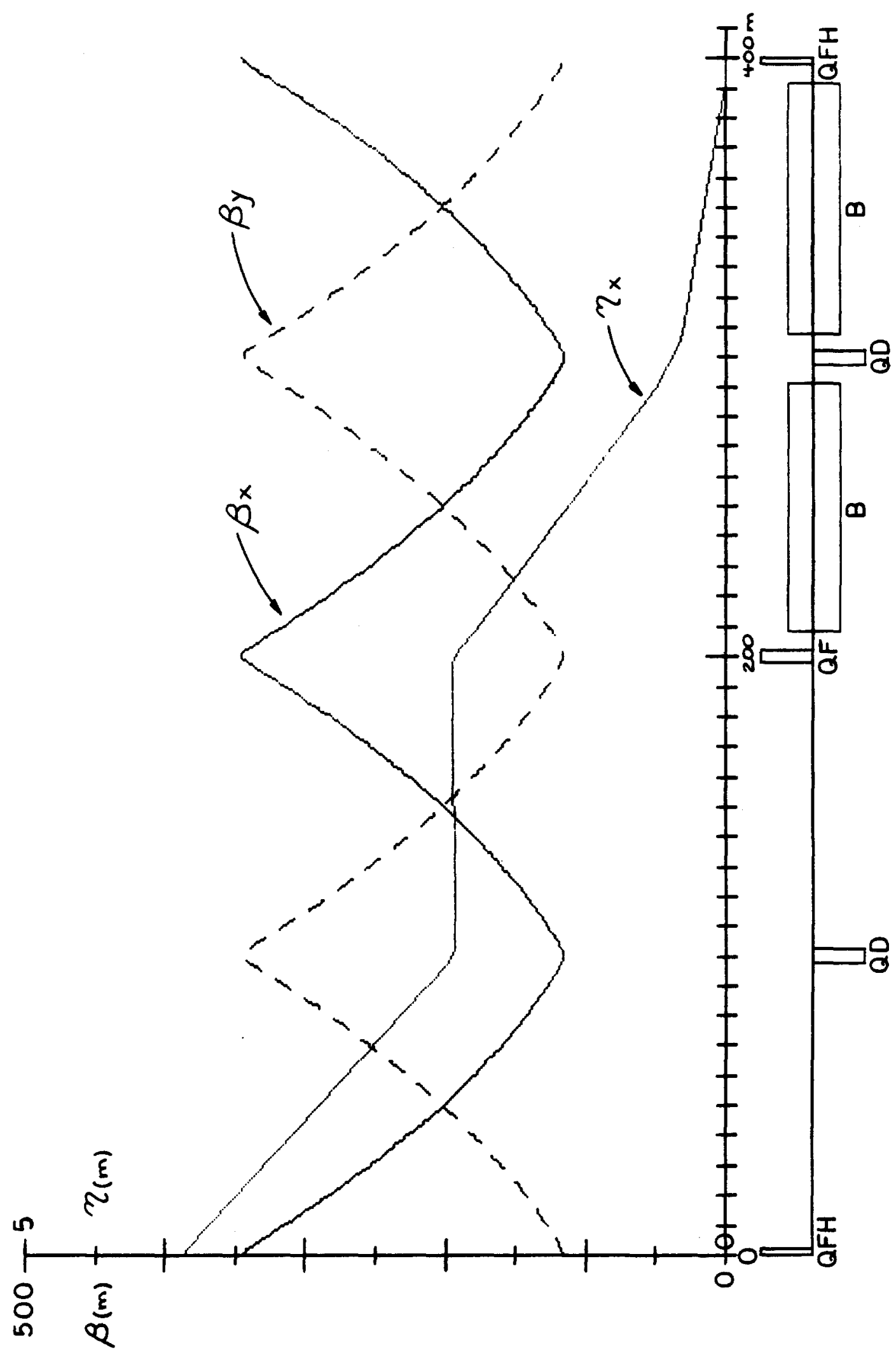

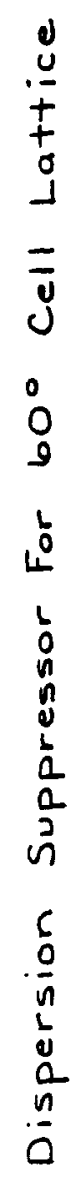
品 


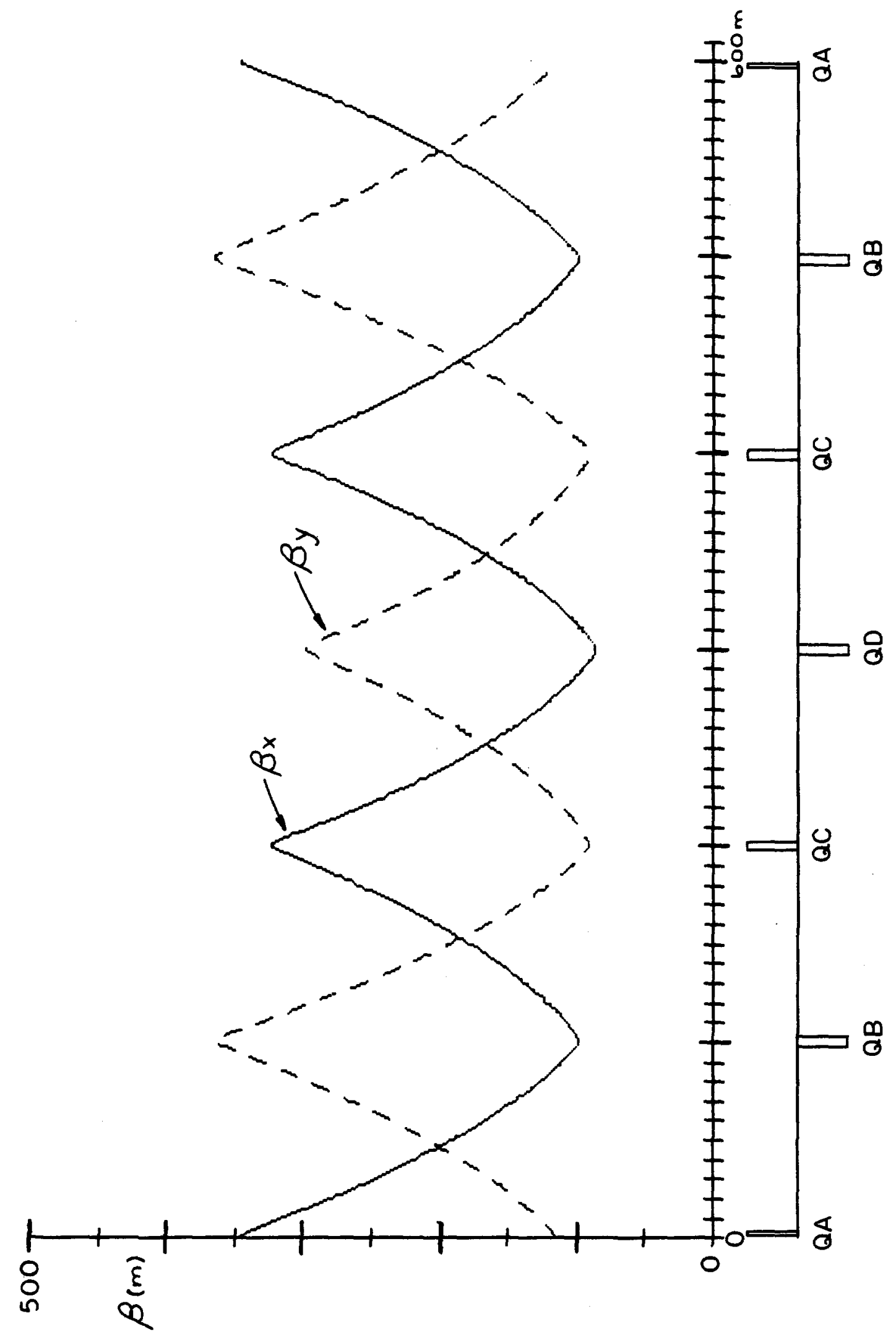

0
6
0
0
5
0
1
1
$\vdots$
1 


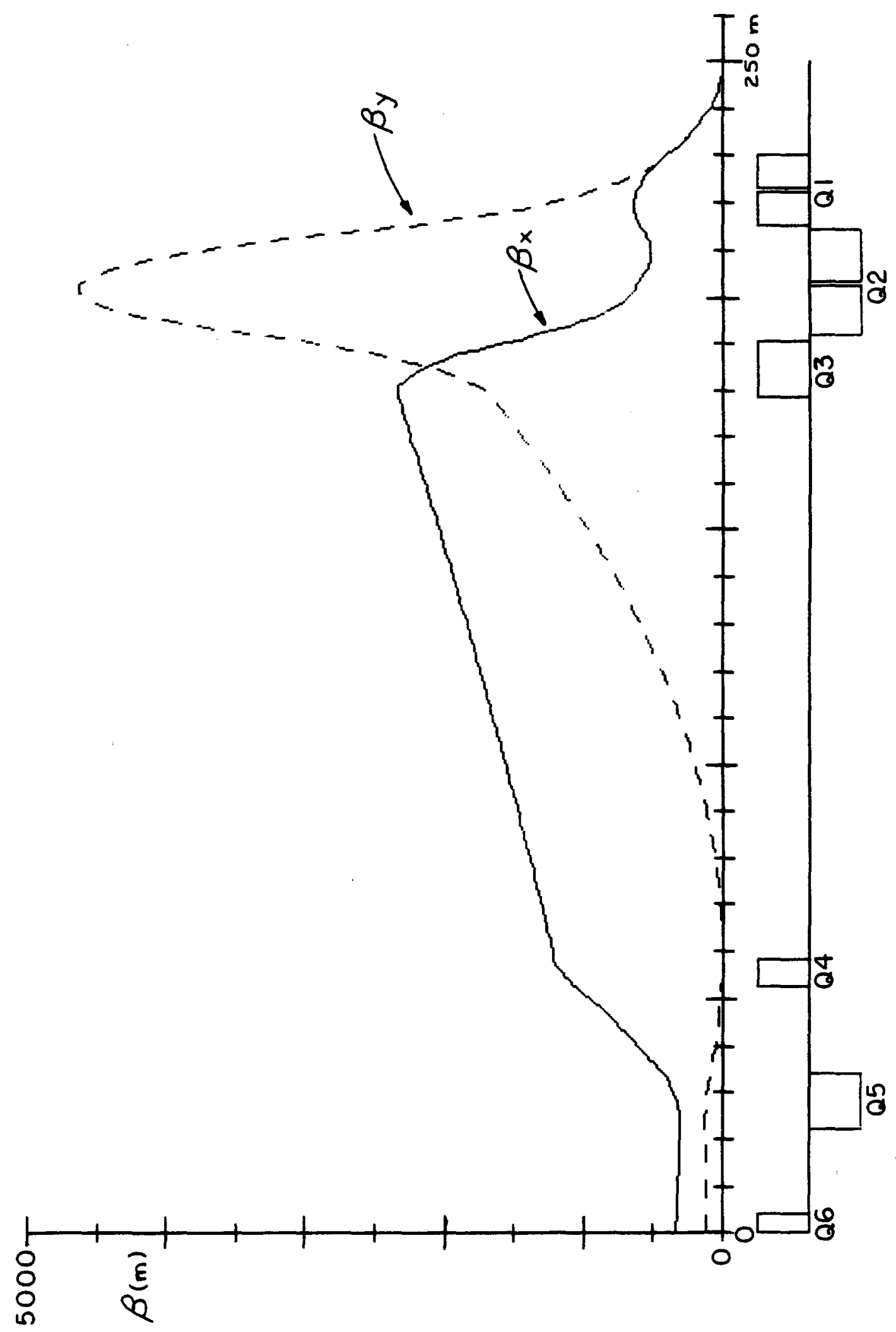

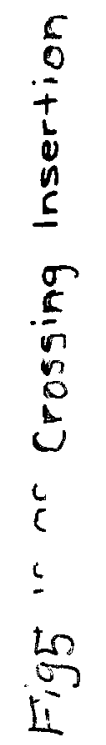




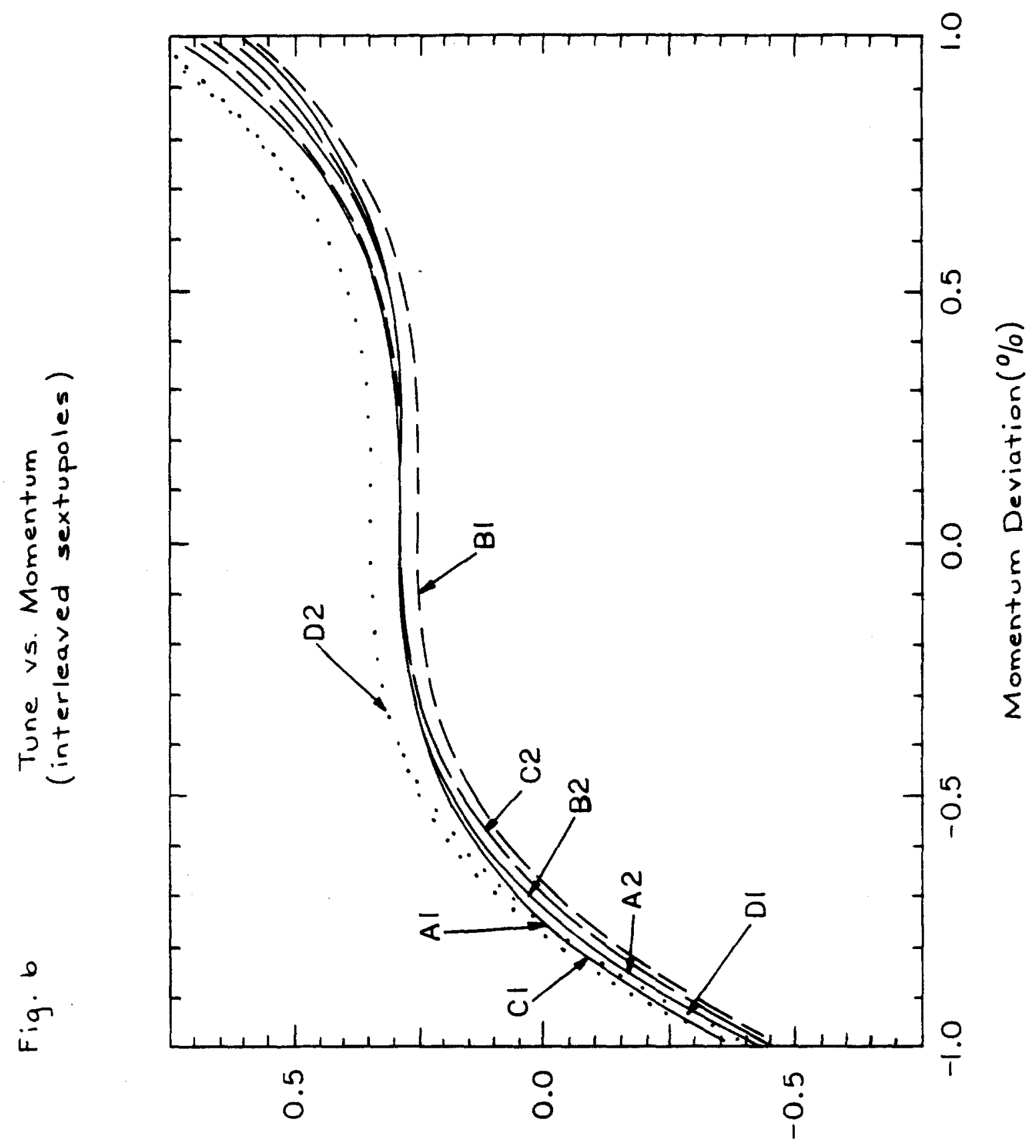

ounl 1000!tood 
Fig. 7 Interaction Point Betas vs. Momentum

(interleaved sextupoles)

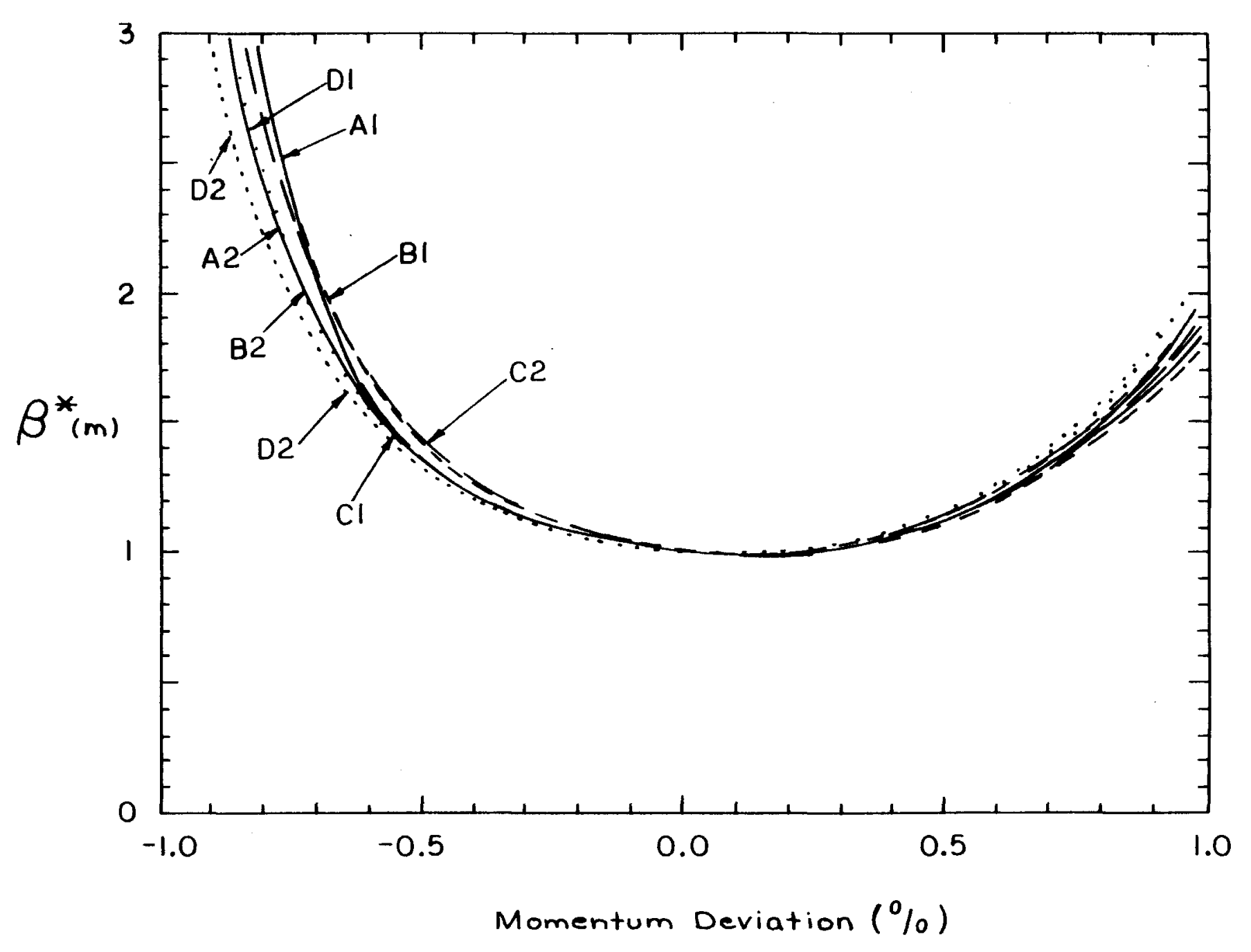




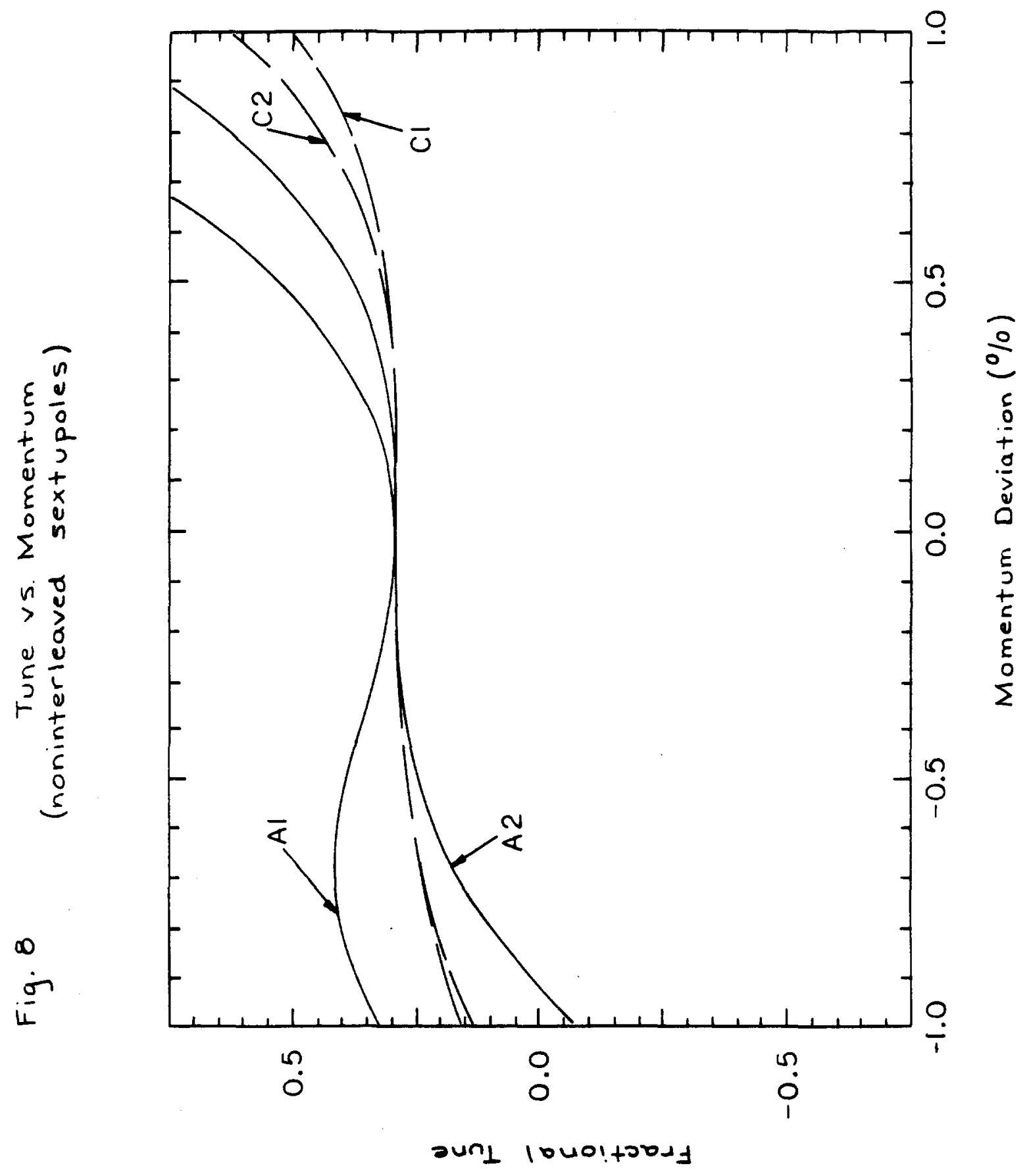




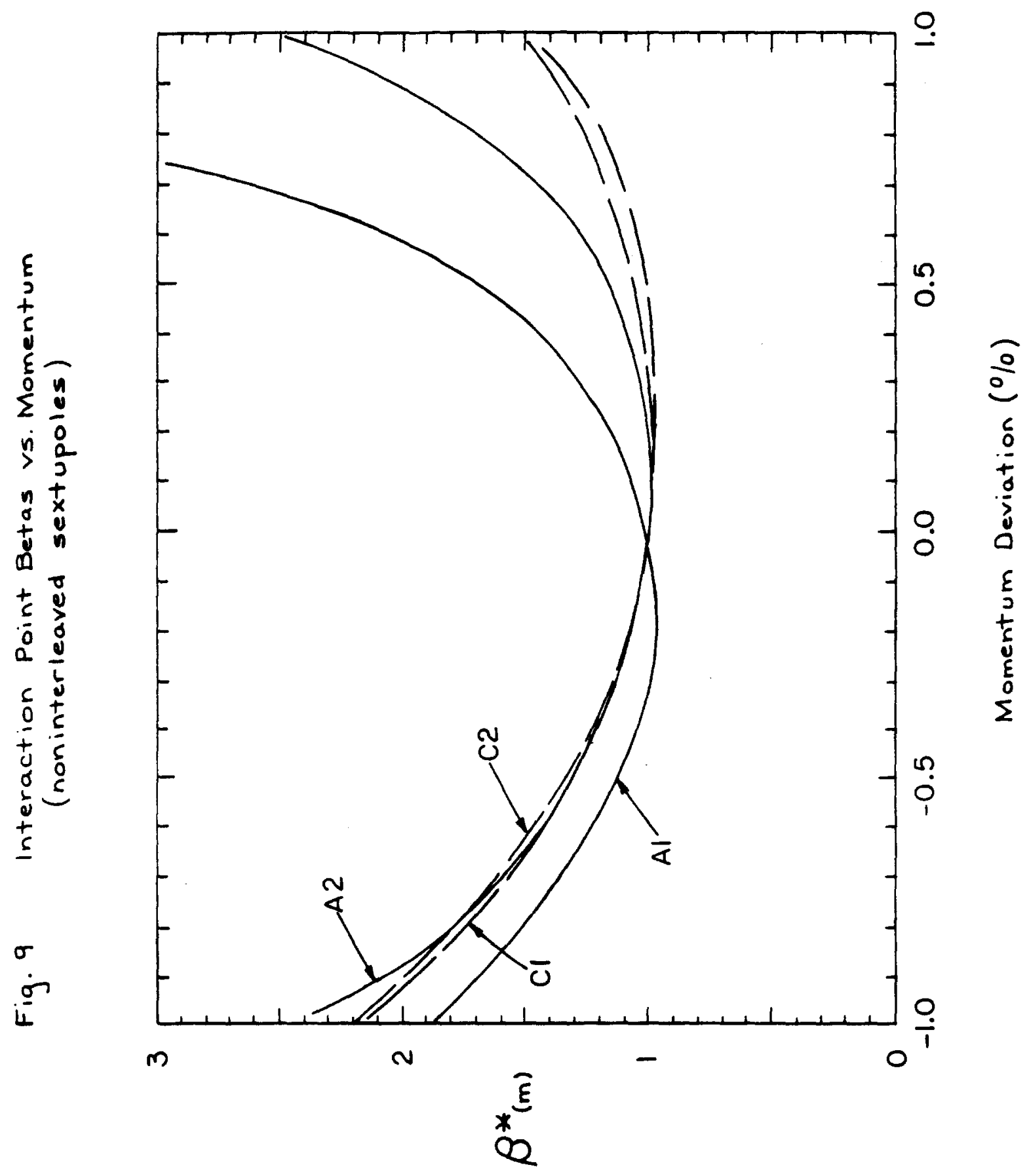

